\title{
Resveratrol attenuates acute kidney injury by inhibiting death receptor-mediated apoptotic pathways in a cisplatin-induced rat model
}

\author{
QIUFA HAO $^{1 *}$, XIAOYAN XIAO ${ }^{*}$, JUNHUI ZHEN $^{2}$, JINBO FENG $^{3}$, CHUN SONG $^{4}$, BEI JIANG $^{1}$ and ZHAO HU $^{1}$ \\ Departments of ${ }^{1}$ Nephrology and ${ }^{2}$ Pathology; ${ }^{3}$ Institute of Obstetrics and Gynecology, Qilu Hospital of Shandong University; \\ ${ }^{4}$ Department of Pharmacy, Shandong University School of Pharmacy, Jinan, Shandong 250012, P.R. China
}

Received October 14, 2015; Accepted August 3, 2016

DOI: $10.3892 / \mathrm{mmr} .2016 .5714$

\begin{abstract}
Acute kidney injury is a clinical syndrome characterized by a loss of renal function and acute tubular necrosis. Resveratrol exerts a wide range of pharmacological effects based on its anti-inflammatory, antioxidant and cytoprotective properties. The present study aimed to evaluate whether resveratrol attenuates acute kidney injury in a cisplatin-induced rat model and to investigate the potential mechanisms involved. Rats were randomly divided into four treatment groups: Control, cisplatin, resveratrol, and cisplatin plus resveratrol. Rats exposed to cisplatin displayed acute kidney injury, identified by analysis of renal function and histopathological observation. Resveratrol significantly ameliorated the increased serum creatinine, blood urea nitrogen, renal index and histopathological damage induced by cisplatin. Furthermore, compared with untreated control animals, cisplatin lead to significantly increased expression of Fas ligand, tumor necrosis factor- $\alpha$ (TNF- $\alpha$ ), caspase- 8 and Bcl-2 associated protein $\mathrm{X}$ apoptosis regulator (Bax), and decreased expression of anti-apoptosis regulators, $\mathrm{BH} 3$ interacting domain death agonist (BID) and $\mathrm{B}$ cell lymphoma 2 apoptosis regulator (Bcl-2). Administration of resveratrol significantly reversed the cisplatin-induced alteration in these apoptosis-associated proteins. In conclusion, these findings suggest that resveratrol attenuates cisplatin-induced acute kidney injury through inactivation of the death receptor-mediated apoptotic pathway, and may provide a new therapeutic strategy to ameliorate the process of acute kidney injury.
\end{abstract}

Correspondence to: Dr Zhao Hu, Department of Nephrology, Qilu Hospital of Shandong University, 107 Wenhua Xi Road, Jinan, Shandong 250012, P.R. China

E-mail: hz12040813@163.com

*Contributed equally

Key words: apoptosis, cisplatin, death receptor-mediated apoptotic pathways, resveratrol, acute kidney injury

\section{Introduction}

Acute kidney injury (AKI) is a multifactorial and multiphasic clinical syndrome, characterized by an abrupt (hours to days) reduction in renal function, together with an accumulation of metabolic waste and toxins, such as serum creatinine (Scr) and blood urea nitrogen (BUN), and/or decreased urine output $(1,2)$. It is estimated that AKI affects $1.9 \%$ of all hospital inpatients, including $>60 \%$ of patients in the intensive care unit $(2,3)$. The physiopathological mechanism of AKI is characterized by renal tubular damage, vascular injury and inflammation $(1,4)$. Injury and death of tubular cells contributes to the pathogenesis of AKI and apoptosis appears to be crucial during this process (1). Cisplatin (CP), an effective antineoplastic drug, induces a loss of renal function in $25-35 \%$ of patients following a single administration (5). Acute tubular damage caused by $\mathrm{CP}$ occurs primarily in the renal proximal tubular cells, particularly in the S3 segment, due to $\mathrm{CP}$ accumulation in the area $(5,6)$. The pathophysiology of CP-induced acute renal tubular injury is associated with inflammation, oxidative stress and apoptosis (5-7). Via these mechanisms, apoptosis of the renal tubular cells is a key mode of cell death (8).

The extrinsic apoptotic pathway may contribute to tubular cell loss in AKI (1). TNF receptor 1 (TNFR1) and Fas (also known as CD95 or APO-1) are important transmembrane components of the tumor necrosis factor (TNF) family of receptors (9-11), whose ligands are TNF- $\alpha$ and Fas-ligand (Fas-L), respectively $(5,11)$. Following the interaction between TNF family ligands and receptors, the conserved death domains located in the cytoplasmic tails allow recruitment of downstream adaptors, such as TNFR1-associated death domain protein and FAS-associated death domain protein (FADD). The subsequent interaction of FADD with Fas or TNFR1 activates the recruitment of caspase- $8(6,12,13)$. Caspase- 8 directly activates the downstream effector, caspase-3, or cleaves $\mathrm{BH} 3$ interacting domain death agonist (BID), a death-inducing member of the B cell lymphoma 2 (Bcl-2) family (14,15). BID is cleaved to form truncated BID (tBID), which is translocated to the mitochondria and promotes a mitochondrial-dependent apoptotic pathway involving Bcl-2 associated X (Bax) and Bcl-2 (14,16). Previous studies have demonstrated that TNFR1 knockout mice are resistant to $\mathrm{CP}$-induced AKI $(6,12)$. 
However, studies that have investigated the role of TNFR1 in apoptosis in renal tubular cells are limited and preliminary (13). In addition, whether Fas/Fas-L induces apoptosis in renal tubular cells still remains controversial (10). However, high Fas expression levels have been observed in renal tubular cells following acute and chronic renal failure $(17,18)$.

Resveratrol (trans-3,4',5-trihydroxystilbene; RSV) is a polyphenolic phytoalexin present in numerous edible plants, including mulberries, peanuts and grapes (19-21). RSV has been studied in vivo and in vitro $(19,21)$ and has been demonstrated to possess a wide range of pharmacological effects, including cardioprotective (22), neuroprotective (23), nephroprotective (24), antineoplastic (25) and antidiabetic (26) effects, as a result of its anti-inflammatory, antioxidant and cytoprotective properties (19). Previous studies have demonstrated the benefits of RSV towards several types of kidney disease, including diabetic nephropathy (27), drug-induced renal injury $(28,24)$, and ischemia-reperfusion and sepsis-induced kidney injuries $(29,30)$. The present study, therefore, aimed to determine whether RSV attenuates CP-induced AKI in a rat model and to investigate the potential mechanisms of attenuation.

\section{Materials and methods}

Reagents. CP (CAS no. 15663-27-1) and RSV (CAS no. 501-36-0) were purchased from Sigma-Aldrich (St. Louis, MO, USA). Primary antibodies were supplied as follows: Mouse anti-Fas-L (cat. no. sc-19988), mouse anti-Bcl-2 (cat. no. sc-7382), rabbit-anti-Bax (cat. no. sc-6236), rabbit anti-BID (cat. no. sc-11423) from Santa Cruz Biotechnology, Inc. (Santa Cruz, Dallas, TX, USA); rabbit anti-TNF- $\alpha$ (cat. no. ab9755) and rabbit anti-caspase-8 (cat. no. ab181580) from Abcam (Cambridge, UK); and rabbit anti- $\beta$-actin from Merck Millipore (Darmstadt, Germany). Horseradish peroxidase (HRP)-conjugated anti-mouse IgG (cat. no. 12-349) and anti-rabbit IgG secondary antibodies (cat. no. 12-348) were obtained from Merck Millipore. The terminal deoxynucleotidyl transferase dUTP nick-end labeling (TUNEL) in situ cell death detection kit was purchased from Roche Diagnostics GmbH (Mannheim, Germany).

Animals. A total of 28 adult male Wistar rats (weighing 180-200 g; 6-8 weeks old) were obtained from Shandong University Laboratory Animal Center (Jinan, China). Rats were acclimated to laboratory conditions for one week prior to experiments, and were consistently maintained in polycarbonate cages under standard conditions of temperature $\left(20-23^{\circ} \mathrm{C}\right)$ and humidity (50-70\%) with $12 \mathrm{~h}$ light-dark cycles. All rats had unrestricted access to food and water. All experiments involving rats were performed in accordance with the Guidelines for Animal Experiments of Qilu Hospital, Shandong University (Jinan, China) and all experimental procedures were approved by the Institutional Ethics Committee for Laboratory Animal Care of Qilu Hospital, Shandong University.

Animal treament. Rats were randomly divided into four treatment groups, with seven animals per group: i) Control group (NS) received intraperitoneal (ip) injections of $0.9 \%$ saline $(10 \mathrm{ml} / \mathrm{kg})$ on day 1 and day 3 ; ii) RSV group received ip injections of $10 \mathrm{mg} / \mathrm{kg} \mathrm{RSV}(2 \mathrm{mg} / \mathrm{ml}$, dissolved in $0.9 \%$ saline) on day 1 and day 3; iii) $\mathrm{CP}$ group received an ip injection of $8 \mathrm{mg} / \mathrm{kg} \mathrm{CP}(1 \mathrm{mg} / \mathrm{ml}$, dissolved in $0.9 \%$ saline $)$ on day 1 and an ip injection of $0.9 \%$ saline $(10 \mathrm{ml} / \mathrm{kg})$ on day 3 ; iv) $\mathrm{CP}+\mathrm{RSV}$ group received ip injections of $8 \mathrm{mg} / \mathrm{kg} \mathrm{CP}$ ( $1 \mathrm{mg} / \mathrm{ml}$, dissolved in $0.9 \%$ saline) followed $30 \mathrm{~min}$ later by $10 \mathrm{mg} / \mathrm{kg} \mathrm{RSV}$ ( $2 \mathrm{mg} / \mathrm{ml}$, dissolved in $0.9 \%$ saline) on day 1 , and an ip injection of $10 \mathrm{mg} / \mathrm{kg} \mathrm{RSV}(2 \mathrm{mg} / \mathrm{ml}$, dissolved in $0.9 \%$ saline) on day 3 .

On day 5 of treatment, rats were first weighed before they were anesthetized by intraperitoneal injection of $10 \%$ chloral hydrate ( $0.4 \mathrm{ml} / 100 \mathrm{~g}$ body weight; CAS no. 302-17-0; Damao Chemical Reagent Factory, Tianjin, China), and sacrificed by bloodletting from the left ventricle. Serum was separated from the blood by centrifugation $\left(1,500 \mathrm{x}\right.$ g at $4^{\circ} \mathrm{C}$ for $\left.15 \mathrm{~min}\right)$ and immediately stored at $-20^{\circ} \mathrm{C}$ prior to analysis. Rat kidneys were perfused in situ through the left ventricle with $0.9 \%$ saline, then excised, weighed and cut in half by coronal position. Half of each kidney was immediately stored at $-80^{\circ} \mathrm{C}$, and half immersed in $4 \%$ paraformaldehyde buffered with phosphate-buffered saline (PBS) at $4^{\circ} \mathrm{C}$, then fixed for $24 \mathrm{~h}$ and embedded in paraffin.

Assessment of renal function. Measurements of Scr and BUN were conducted in Qilu Hospital of Shandong University by a Cobas $^{\circledR} 8000$ modular analyzer (Roche Diagnostics GmbH). The renal index (RI) was calculated as follows: Both kidney weights (g) / animal weight (g) x 1,000.

Histopathological observation. Paraffinized kidneys were cut into 3-5 $\mu \mathrm{m}$-thick sections, before they were deparaffinized and stained with hematoxylin and eosin (H\&E; Nanjing Jiancheng Bioengineering Institute, Nanjing, China). Sections were immersed in $0.5 \%$ eosin for $1 \mathrm{~min}$ and $0.1 \%$ hematoxylin for $5 \mathrm{~min}$. The tubular damage score assessment was performed in 10 fields of 5 sections per group using the following index of renal tubular necrosis: Score of 0 (absence of damage); score of 1 ( $<25 \%$ damage); score of 2 (25-50\% damage); score of 3 (50-75\% damage); and score of 4 ( $>75 \%$ damage).

Immunohistochemistry. Tissue sections underwent deparaffinization and rehydration with xylene and 100, 95, 90 and $80 \%$ ethanol gradients. Following 3 washes with PBS, slices were placed in $0.01 \%$ sodium citrate buffer $(\mathrm{pH} 6.0)$, and heated by microwave $\left(10 \mathrm{~min}\right.$ at $\left.93-95^{\circ} \mathrm{C}\right)$ for antigen retrieval. Tissue sections were then cooled, washed with PBS 3 times and immersed in $0.1 \%$ Triton X-100 for $15 \mathrm{~min}$. Sections were incubated with $3 \%$ hydrogen peroxide for $10 \mathrm{~min}$ at $15-25^{\circ} \mathrm{C}$ in the dark, to block endogenous peroxidase activity, followed by incubation with $10 \%$ goat serum (Nanjing Jiancheng Bioengineering Institute) for $45 \mathrm{~min}$ at $37^{\circ} \mathrm{C}$, and then with mouse anti-Fas-L (dilution, 1:200) rabbit anti-Bax (dilution, 1:200) and mouse anti-Bcl-2 (dilution, 1:200) primary antibodies at $4^{\circ} \mathrm{C}$ overnight. The negative control sections were treated with PBS. Sections were subsequently washed and incubated with polymer helper for $20 \mathrm{~min}$ at $37^{\circ} \mathrm{C}$, together with HRP-labeled anti-rabbit IgG polymer (cat. no. PV-9001) or HRP-labeled anti-mouse IgG polymer (cat. no. PV-9002) from the Polink-2 plus ${ }^{\circledR}$ Polymer HRP Detection System (Beijing Zhongshan Golden Bridge Biotechnology Co., Ltd., Beijing, China). After washing 3 times with PBS, tissue slices 
Table I. Effects of RSV on levels of Scr, BUN and RI.

\begin{tabular}{lcccc}
\hline Group & $\mathrm{n}$ & Scr $(\mu \mathrm{mol} / \mathrm{l})$ & BUN $(\mathrm{mmol} / \mathrm{l})$ & $\mathrm{RI}$ \\
\hline NS & 7 & $31.14 \pm 6.26$ & $7.42 \pm 1.21$ & $7.99 \pm 0.12$ \\
RSV & 7 & $28.71 \pm 5.28$ & $6.13 \pm 0.61$ & $8.00 \pm 0.16$ \\
CP & 7 & $192.29 \pm 11.44^{\mathrm{a}}$ & $43.39 \pm 2.20^{\mathrm{a}}$ & $9.36 \pm 0.11^{\mathrm{a}}$ \\
CP+RSV & 7 & $63.86 \pm 8.71^{\mathrm{b}}$ & $19.04 \pm 1.94^{\mathrm{b}}$ & $8.81 \pm 0.17^{\mathrm{b}}$
\end{tabular}

Values are presented as the mean \pm standard deviation. ${ }^{\text {a }}<0.05$ vs. NS group; ${ }^{b} \mathrm{P}<0.05$ vs. CP group. RSV, resveratrol group; Scr, serum creatinine; BUN, blood urea nitrogen; RI, renal index; n, number of rats per group; NS, control group; CP, cisplatin group.

were stained with 3,3'-diaminobenzidine solution (DAB; cat. no.ZLI-9017; Beijing Zhongshan Golden Bridge Biotechnology Co., Ltd). Tissue slices were subsequently stained with $0.1 \%$ hematoxylin for $5 \mathrm{~min}$. The stained slides were observed under a light microscope, and brown areas were deemed as positively stained. High power fields ( $\mathrm{n}=10$ per section) were randomly selected and 2 sections/group were scored. The intensity of dye color was graded as follows: Score 0 , no color; score 1 light yellow; score 2, light brown; and score 3 , brown. The percentage of positive areas was graded as follows: Score 0 , $<5 \%$; score 1, 5-25\%; score 2, 25-50\%; score 3, 50-75\%; and score $4,>75 \%$. The two parameters were added to produce a final score.

TUNEL assay. The in situ cell death detection kit was used to detect cell apoptosis. TUNEL assay was performed according to the manufacturer's instructions. Briefly, deparaffinized tissue sections were incubated with $3 \%$ hydrogen peroxide in methanol $\left(10 \mathrm{~min}\right.$ at $15-25^{\circ} \mathrm{C}$ in the dark), washed 3 times with PBS, then incubated with $0.1 \%$ Triton X-100 in freshly prepared $0.01 \%$ sodium citrate $\left(8 \mathrm{~min}\right.$ at $\left.25^{\circ} \mathrm{C}\right)$. Tissue sections were incubated with enzyme and labeling solutions (1:9; $60 \mathrm{~min}$ at $\left.37^{\circ} \mathrm{C}\right)$. Following 3 washes in PBS, slices were stained with DAB and hematoxylin. Negative controls were incubated with labeling solution only. TUNEL positive nuclei were counted in 10 random, non-overlapping high power fields of 2 tissue sections.

Western blot. Perfused kidney tissue was snap frozen in liquid nitrogen, and $30 \mathrm{mg}$ of kidney tissue was homogenized with radioimmunoprecipitation assay buffer. The supernatant was collected for protein quantification following centrifugation at $15,000 \mathrm{x} \mathrm{g}$ for $30 \mathrm{~min}$ at $4^{\circ} \mathrm{C}$. The concentration of protein extracted from rat kidney tissue samples was quantified using a Bicinchoninic Acid Protein assay kit (Beyotime Institute of Biotechnology, Jiangsu, China). A total of $30 \mu \mathrm{g}$ protein from each sample was separated on $12 \%$ gels by sodium dodecyl sulfate-polyacrylamide gel electrophoresis and subsequently electroblotted onto polyvinylidene difluoride membranes. Membranes were incubated with blocking buffer (5\% skimmed milk) at room temperature for $60 \mathrm{~min}$ and then incubated with primary antibodies (mouse anti-Fas-L, 1:200; rabbit anti-BID, 1:200; rabbit anti-Bax, 1:200; mouse anti-Bcl-2, 1:200; rabbit anti-TNF- $\alpha, 1: 1,000$; rabbit anti-caspase-8, 1:1,000; and rabbit anti- $\beta$-actin, $1: 500)$ at $4^{\circ} \mathrm{C}$ overnight. Following washing in Tris-buffered saline with $0.1 \%$ Tween 20 (TBST), membranes were incubated with anti-mouse or anti-rabbit secondary antibodies (dilution, 1:5,000) at room temperature for $60 \mathrm{~min}$. Membranes were then washed in TBST and the blots were detected using the Immobilon ${ }^{\mathrm{TM}}$ Western Chemiluminescent HRP Substrate (EMD Millipore, Billerica, MA, USA) followed by autoradiography. Protein density for quantification was determined using Image J software (version 1.45; National Institutes of Health, Bethesda, MD, USA). $\beta$-actin was used as a loading control and relative quantities of all proteins were expressed as a ratio to that of the NS group.

Statistical analysis. Data are expressed as the mean \pm standard deviation. Analyses were performed using one-way analysis of variance with SPSS 19.0 software (SPSS Inc., Chicago, IL, USA). The least-significant difference post-hoc test was used to assess differences between two groups. $\mathrm{P}<0.05$ was considered to indicate a statistically significant difference.

\section{Results}

Effects of $R S V$ on renal function in $C P$-induced $A K I$. It is known that typical AKI is characterized by a significant increase in Scr levels (2). As presented in Table I, CP led to significant increases in the levels of Scr, BUN and RI compared with the control group $(\mathrm{P}<0.001)$. However, co-treatment of RSV and $\mathrm{CP}$ attenuated the increase of Scr, BUN and RI compared with the CP group $(\mathrm{P}<0.001)$.

Renoprotective and anti-apoptotic effects of RSV in $C P$-induced $A K I$. H\&E staining of kidney tissues was used to evaluate the histological changes in renal tubular epithelium. As presented in Fig. 1A, histological examination revealed that the kidneys from the control and RSV groups maintained normal tubular morphology, whereas the kidneys from the $\mathrm{CP}$ group displayed significant features of AKI, including brush border loss, epithelial cell vacuolation and cast formation. Semi-quantitative analysis of the extent of the histological damage in the $\mathrm{CP}$ group was scored as 3.04, in comparison with no damage in the control group $(\mathrm{P}<0.001$; Fig. 1B). By contrast, the extent of tubular damage was significantly reduced in the $\mathrm{CP}+\mathrm{RSV}$ group compared with the $\mathrm{CP}$ group, with a histological damage score of $1.9(\mathrm{P}<0.001$; Fig. 1A and $\mathrm{B})$.

Tubular cell apoptosis was detected in the CP-induced AKI model by TUNEL assay, as presented in Fig. 1C. The NS and RSV groups exhibited limited apoptosis (Fig. 1D), whereas 

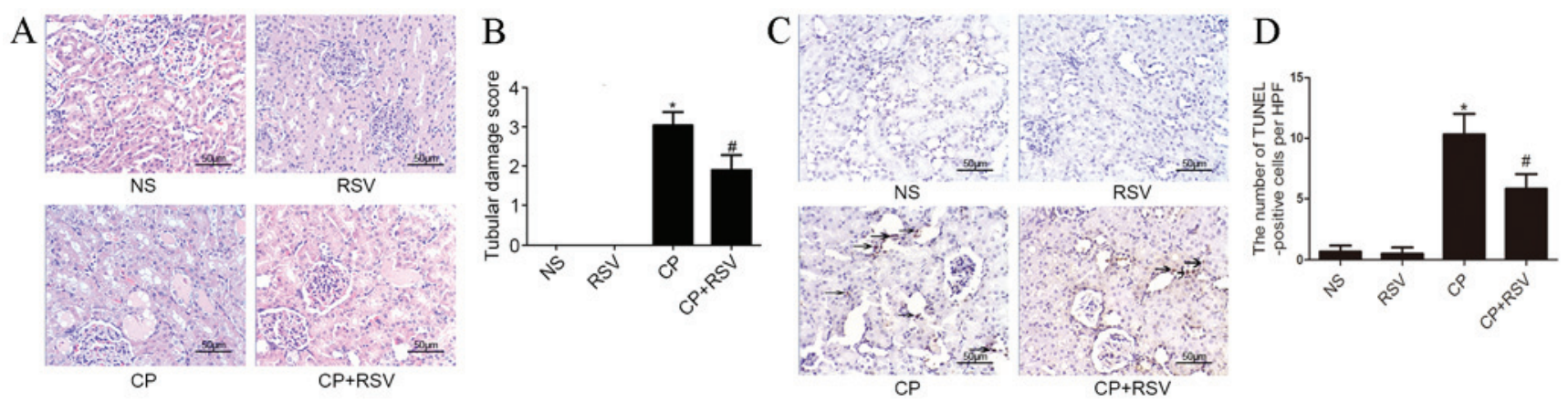

Figure 1. Renoprotective and anti-apoptotic effects of RSV in CP-induced acute kidney injury. (A) Representative images of hematoxylin and eosin staining (original magnification, $\mathrm{x} 400$ ) and (B) tubular damage score of kidney sections. (C) TUNEL staining of kidney sections, with arrows on the panel indicating TUNEL-positive cells (original magnification, x400). (D) Quantification of TUNEL-positive cells. Values are presented as the mean \pm standard deviation of three independent experiments. " $\mathrm{P}<0.05$ vs. NS group; ${ }^{\text {P }}<0.05$ vs. CP group. NS, untreated control; RSV, resveratrol; CP, cisplatin; TUNEL, terminal deoxynucleotidyl transferase dUTP nick-end labeling; HPF, high-power field.
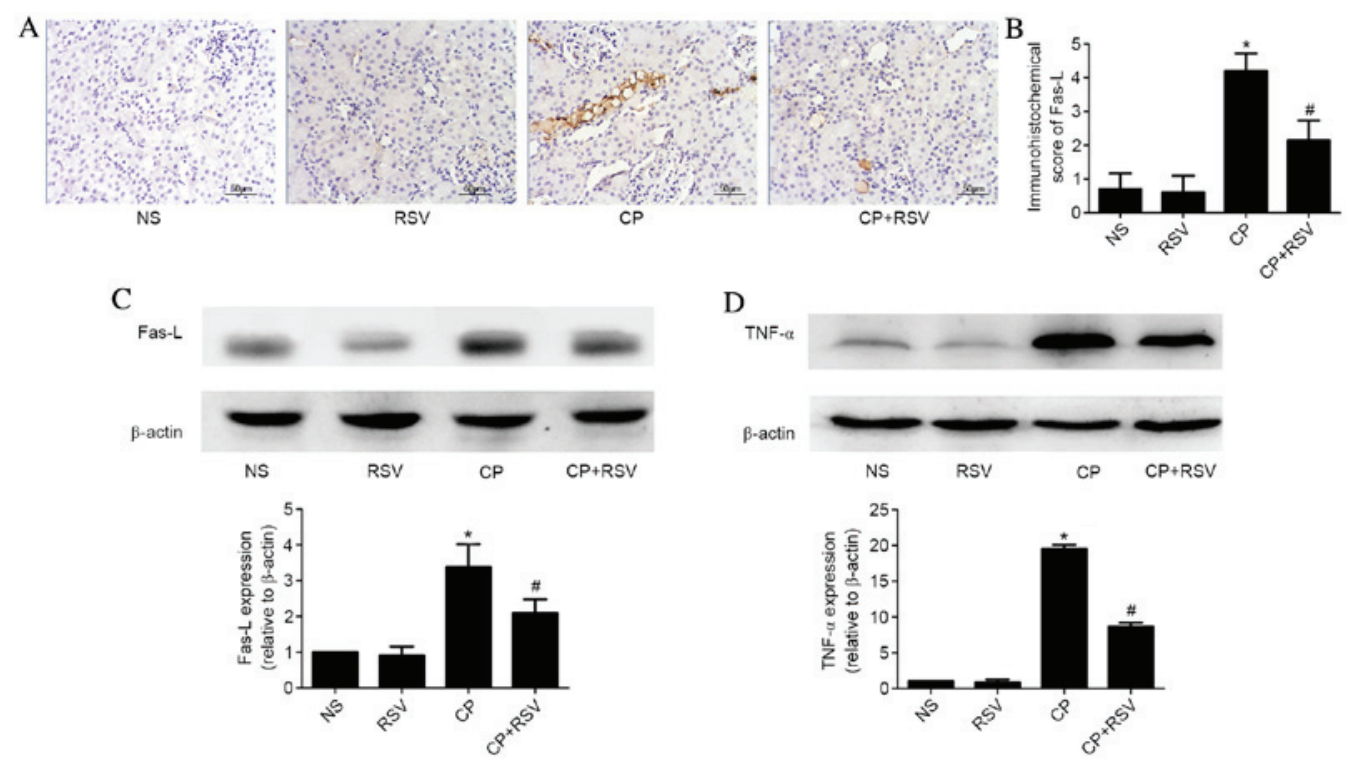

Figure 2. Western blot analysis and immunohistochemistry of Fas-L and TNF- $\alpha$. (A) Expression of Fas-L detected in damaged tubules by immunohistochemistry (original magnification, $\mathrm{x} 400$ ) and (B) quantification of Fas-L in kidney sections. Western blot analysis and quantification of protein expression levels of (C) Fas-L and (D) TNF- $\alpha$, relative to $\beta$-actin control. Values are presented as the mean \pm standard deviation of three independent experiments. " $\mathrm{P}<0.05$ vs. NS group; ${ }^{\#} \mathrm{P}<0.05$ vs. CP group. NS, untreated control; RSV, resveratrol; CP, cisplatin; Fas-L, Fas ligand; TNF- $\alpha$, tumor necrosis factor- $\alpha$.

the number of TUNEL-positive nuclei, indicating apoptosed cells, was significantly increased in the $\mathrm{CP}$ group compared with the NS group $(\mathrm{P}<0.001$; Fig. 1D). However, the number of TUNEL-positive cells in the CP+RSV group was significantly reduced compared with the $\mathrm{CP}$ group $(\mathrm{P}<0.001)$, indicating reduced apoptosis in this group.

Effect of RSV on vital protein expressions in the process of $C P$-induced AKI. Death receptor-mediated apoptotic pathways have been implicated in CP-induced AKI. Thus, the expression of certain vital proteins in this signaling pathway, including Fas-L, TNF- $\alpha$ and caspase-8, were examined. The function of caspase- 8 in the intrinsic mitochondrial apoptotic pathway was also investigated by examining the expression levels of BID, Bax and Bcl-2.

As presented in Fig. 2A and B, Fas-L staining score in the damaged tubules was significantly higher in the $\mathrm{CP}$ group compared with the NS group $(\mathrm{P}<0.001)$, and significantly reduced in the $\mathrm{CP}+\mathrm{RSV}$ group compared with the $\mathrm{CP}$ group $(\mathrm{P}<0.001)$. Western blot analysis of Fas-L protein expression was consistent with the immunohistochemical observations (NS vs. $\mathrm{CP}, \mathrm{P}<0.001$; $\mathrm{CP}$ vs. $\mathrm{CP}+\mathrm{RSV}, \mathrm{P}=0.004$; Fig. $2 \mathrm{C}$ ). Similarly, TNF- $\alpha$ expression was significantly higher in the CP group compared with the NS group $(\mathrm{P}<0.001)$, whereas the $\mathrm{CP}+\mathrm{RSV}$ group demonstrated significantly less expression of TNF- $\alpha$ compared with the CP group ( $\mathrm{P}<0.001$; Fig. $2 \mathrm{D})$.

In addition, the expression of caspase- 8 significantly increased in the $\mathrm{CP}$ group compared with the NS group $(\mathrm{P}<0.001$; Fig. $3 \mathrm{~A})$, while the $\mathrm{CP}+\mathrm{RSV}$ group exhibited significantly less expression than the $\mathrm{CP}$ group $(\mathrm{P}<0.001)$. Activated caspase- 8 is known to cleave BID, and thus, may cause the significantly reduced level of BID detected by western blot analysis in the CP group compared with the NS group $(\mathrm{P}<0.001$; Fig. 3B). By contrast, treatment with RSV suppressed this downregulation of BID protein levels in the $\mathrm{CP}+\mathrm{RSV}$ group compared with the $\mathrm{CP}$ group ( $\mathrm{P}=0.005$; Fig. $3 \mathrm{~B})$. 

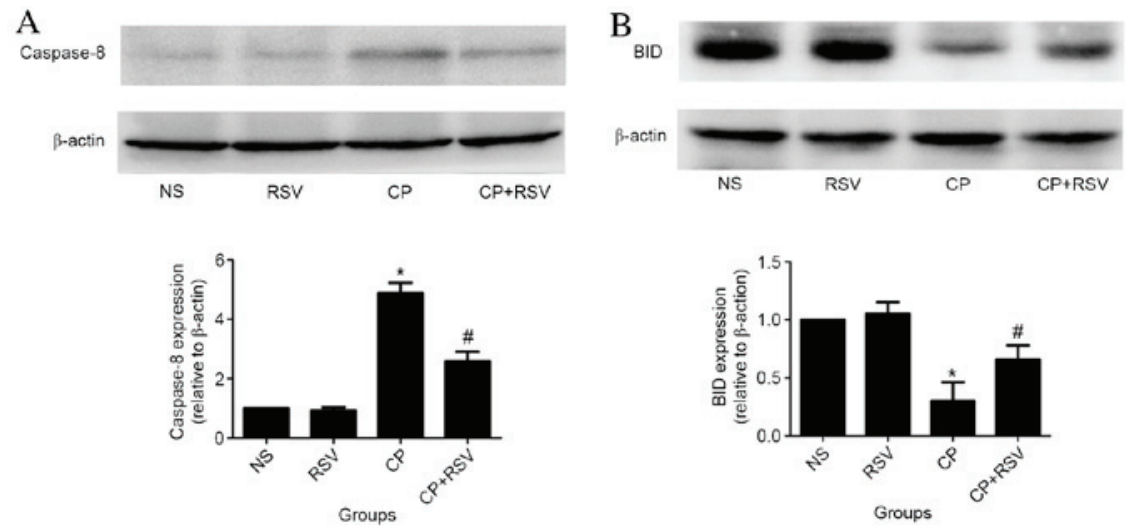

Figure 3. Protein expression levels of caspase-8 and BID. Western blot analysis and quantification protein expression levels of (A) caspase- 8 and (B) BID, relative to $\beta$-actin control. Values are presented as the mean \pm standard deviation of three independent experiments. ${ }^{*} \mathrm{P}<0.05$ vs. $\mathrm{NS}$ group; ${ }^{*} \mathrm{P}<0.05 \mathrm{vs}$. $\mathrm{CP}$ group. NS, untreated control; RSV, resveratrol; CP, cisplatin; BID, BH3 interacting domain death agonist.

Furthermore, expression of the Bax apoptosis regulator was measured by immunohistochemistry and western blotting (Fig. 4). Significantly increased levels of Bax protein expression were observed in the $\mathrm{CP}$ group compared with the NS group by immunohistochemistry $(\mathrm{P}<0.001$; Fig. $4 \mathrm{~A}$ and $\mathrm{B})$ and western blot $(\mathrm{P}<0.001$; Fig $4 \mathrm{C})$, while Bax expression was significantly lower in the $\mathrm{CP}+\mathrm{RSV}$ group than in the $\mathrm{CP}$ group as demonstrated by both immunohistochemistry $(\mathrm{P}<0.001$; Fig. 4A and B) and western blot ( $\mathrm{P}=0.002$; Fig $4 \mathrm{C})$. Expression of the anti-apoptosis regulator Bcl-2 was significantly reduced in the $\mathrm{CP}$ group compared with the NS group as demonstrated by immunohistochemistry $(\mathrm{P}<0.001$; Fig. $4 \mathrm{D}$ and $\mathrm{E})$ and western blot analysis ( $\mathrm{P}=0.004$; Fig. 4F). Expression of $\mathrm{Bcl}-2$ detected in kidneys from the $\mathrm{CP}+\mathrm{RSV}$ group was significantly increased compared with the $\mathrm{CP}$ group as demonstrated by immunohistochemistry ( $\mathrm{P}<0.001$; Fig. $4 \mathrm{D}$ and $\mathrm{E})$ and western blot analysis $(\mathrm{P}<0.001$; Fig. 4F $)$.

\section{Discussion}

The present study sought to investigate whether RSV exerted a renoprotective effect on $\mathrm{CP}$-induced $\mathrm{AKI}$ in a rat model by inhibiting death receptor-mediated apoptotic pathways. RSV was revealed to facilitate a significant reduction in Scr, BUN and RI levels, indicating significantly reduced renal injury and dysfunction which was further verified by H\&E staining and apoptosis analysis of renal histology. In addition, RSV was demonstrated to suppress the upregulation of Fas-L and TNF- $\alpha$, and to modulate the expression levels of the downstream signaling effectors, caspase- 8 , BID, Bax and Bcl-2, to accomplish its protective effect.

As a polyphenolic phytoalexin, RSV has been reported to be beneficial for the prevention of numerous types of kidney disease, including diabetic nephropathy (27), drug-induced renal injury $(28,24)$, and ischemia-reperfusion and sepsis-induced kidney injuries $(29,30)$. Consistent with these observations, the results of the present study demonstrate the beneficial effects of RSV in the prevention of renal tubular damage and dysfunction. The protective effects of RSV were confirmed to be associated with a reduction in Fas-L, TNF- $\alpha$ and caspase- 8 expression levels, which were augmented by CP. Previous studies have reported that RSV is involved in mediating numerous signaling pathways in AKI $(20,28,24)$, however, no study conducted to date has demonstrated the role of RSV in regulating extrinsic apoptotic signaling pathways.

In the present study, it remains unclear whether the positive effects of RSV in preventing damage to renal tubular cells, is directly associated with a reduction in the expression levels of Fas-L, TNF- $\alpha$ and caspase-8. CP-induced tubular apoptosis was initiated by Fas-L, which is expressed on renal tubular cells and immune cells, and is capable of inducing adjacent tubule cell death. Therefore, inhibiting Fas-L may reduce $\mathrm{CP}$-induced tubular apoptosis and completely restore the survival of mice treated with a lethal CP dose (11). TNF- $\alpha$ knockout mice exhibit reduced renal dysfunction, renal histological injury and serum TNF- $\alpha$ levels (31). TNF- $\alpha$ receptor knockout mice also consistently present less renal tubular cell death, further supporting the involvement of death receptor-mediated pathways in the pathogenesis of AKI induced by $\mathrm{CP}$ (11). And the present study demonstrated that CP increased Fas-L, TNF- $\alpha$ and caspase- 8 expression in an AKI model, and that RSV suppressed the upregulation of these effectors. High expression of Fas-L and TNF- $\alpha$ indicates the activation of the extrinsic apoptotic pathway that can be caused by CP. With the interaction between TNF family ligands and their receptors, the downstream signaling molecule, caspase- 8 is also activated, which is a marker of the extrinsic apoptotic pathways (32). It has been demonstrated that RSV can serve a protective role in CP-induced renal injury by reducing free radicals (28) and activating sirtuin 1 (24). The results of the present study demonstrate that RSV can decrease Fas-L, TNF- $\alpha$ and caspase- 8 expression, which is similar to the effect of other antioxidants on CP-induced renal injury, such as epigallocatechin-3-gallate (10) and dimethylthiourea (13). Therefore, these findings indicate that the potential mechanism of RSV in the prevention of CP-induced AKI involves extrinsic apoptotic pathways.

The implementation of programmed cell death is enforced by caspase- 8 through two different pathways $(14,33)$. In pathway 1 , the high caspase- 8 concentration directly activates the downstream effector, caspase-3. Caspase- 3 is then cleaved and stimulates apoptosis. In pathway 2, the low caspase- 8 concentration can cleave BID, a death-inducing member of the Bcl-2 family $(14,15)$, rather than directly activating caspase-3 
A

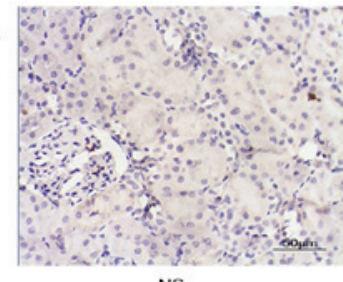

NS

B

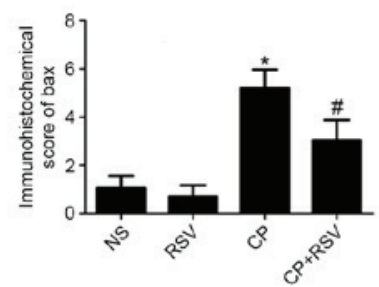

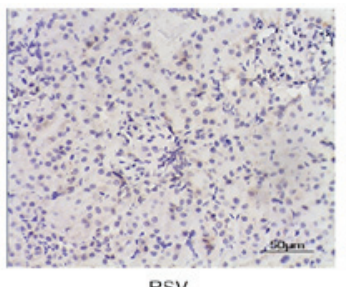

RSV

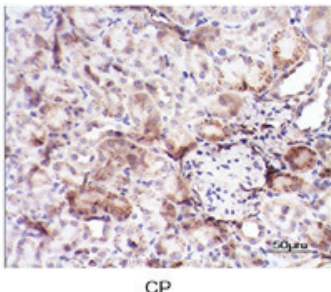

C

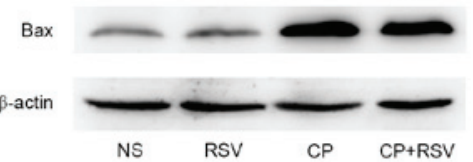

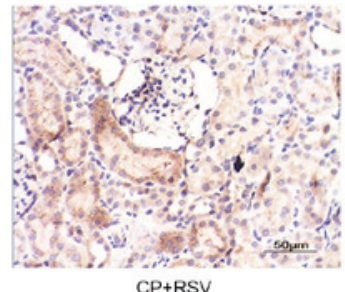

$C P+R S V$

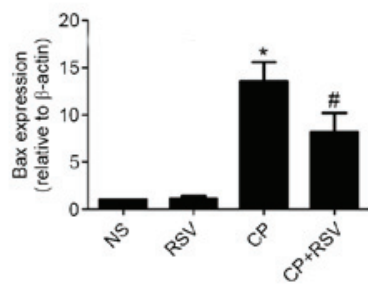

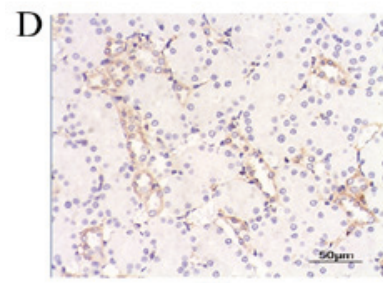

NS
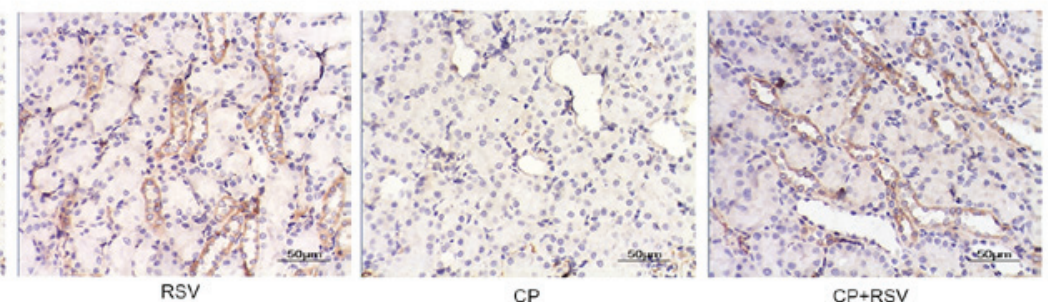

E
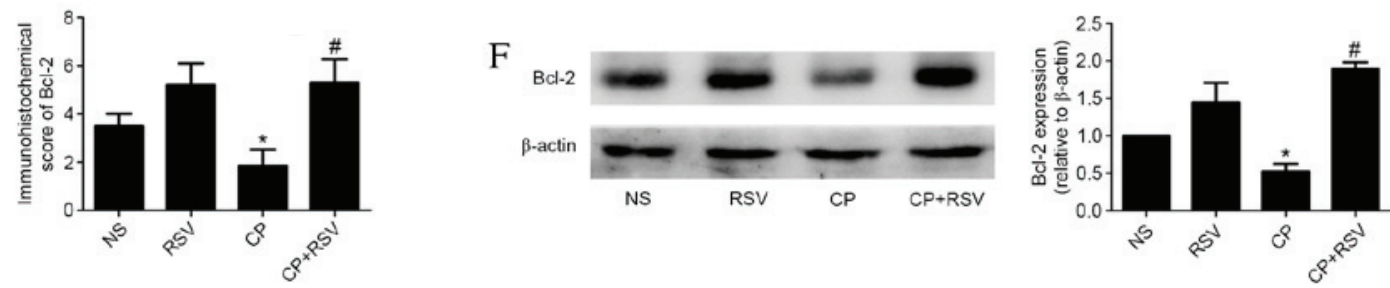

Figure 4. Western blot analysis and immunohistochemistry of Bax and Bcl-2. (A) Immunohistochemical detection of Bax (original magnification, x400) and (B) semi-quantitative scoring of staining. (C) Western blot analysis and quantification of Bax protein levels. (D) Immunohistochemical detection of Bcl-2 (original magnification, x400) and (E) scoring of staining. (F) Western blot analysis and quantification of Bcl-2 protein levels. Values are presented as the mean \pm standard deviation of three independent experiments. ${ }^{*} \mathrm{P}<0.05$ vs. NS group; ${ }^{\text {} P}<0.05$ vs. $\mathrm{CP}$ group. NS, untreated control; RSV, resveratrol; $\mathrm{CP}$, cisplatin; Bax, Bcl-2 associated protein X apoptosis regulator; Bcl-2, B cell lymphoma 2 apoptosis regulator.

to execute programmed cell death. tBID translocates to the mitochondria membrane $(16,32,34)$. Additionally, previous studies have demonstrated that tBID promotes Bax activation and facilitates the insertion/oligomerization of Bax into the mitochondrial outer membrane $(35,36)$. As a result, pores of the mitochondrial membranes are formed, and apoptotic proteins residing in the intermembrane space are released (36). As a member of the anti-apoptotic Bcl-2 family, Bcl-2 can prevent mitochondrial permeability transition of tBID and Bax, and is pivotal for the inhibition of apoptosis $(1,35)$. Overexpression of Bcl-2 suppresses apoptosis $(1,15)$, thus, the cross talk between the mitochondrial and death receptor-mediated apoptotic pathways occurs through caspase- 8 . In the present study, CP has been suggested to increase the cleavage of BID as the expression of BID in the CP group was significantly lower than in the NS group, whereas compared with the CP group the expression of BID increased upon treatment with RSV. Meanwhile, the activation of the intrinsic mitochondrial pathway was examined through examination of Bax and Bcl-2 expression. High level expression of Bax and low level expression of Bcl-2 were observed in the $\mathrm{CP}$ group, and the reverse phenomenon in the CP+RSV group. Previous studies have demonstrated that caspase- 8 (following activation in the extrinsic pathway) can activate the intrinsic apoptosis pathway through Bcl-2 family proteins, such as $\operatorname{Bid}(16,32,34)$. In the present study, $\mathrm{CP}$ is demonstrated to induce the apoptosis pathway mediated by death receptors, which is similar to the effects observed from dimethylthiourea on CP-induced renal injury (13) and the loss of $\alpha(E)$-catenin on CP-challenged renal tubular epithelial cells (15). Finally, the expression of these signal pathways leads to renal tubular cell apoptosis. Therefore, RSV treatment may reduce Bax expression and upregulate BID and Bcl-2 expression via the interaction between the extrinsic and intrinsic signaling pathways through caspase- 8 , which could exert an important protective role in the process of CP-induced AKI.

In conclusion, the present study indicated that RSV may protect against $\mathrm{CP}$-induced $\mathrm{AKI}$, and the underlying mechanism is associated with the suppression of apoptosis via death receptor-mediated pathways. RSV may, therefore, have potential value in the treatment of patients suffering from AKI and warrants further investigation of its therapeutic activity in the clinic. 


\section{Acknowledgements}

The present study is supported by General Financial Grant from China Postdoctoral Science Foundation (no. 2015M572048), and Shandong Province Natural Science Foundation of China (grant no. ZR2014HM037 and ZR2013HM100).

\section{References}

1. Linkermann A, Chen G, Dong G, Kunzendorf U, Krautwald S and Dong Z: Regulated cell death in AKI. J Am Soc Nephrol 25: 2689-2701, 2014

2. Bellomo R, Kellum JA and Ronco C: Acute kidney injury. Lancet 380: 756-766, 2012.

3. Faubel S, Chawla LS, Chertow GM, Goldstein SL, Jaber BL and Liu KD; Acute Kidney Injury Advisory Group of the American Society of Nephrology: Ongoing clinical trials in AKI. Clin J Am Soc Nephrol 7: 861-873, 2012.

4. Lameire NH, Bagga A, Cruz D, De Maeseneer J, Endre Z, Kellum JA, Liu KD, Mehta RL, Pannu N, Van Biesen W and Vanholder R: Acute kidney injury: An increasing global concern. Lancet 382: 170-179, 2013.

5. dos Santos NA, Carvalho Rodrigues MA, Martins NM and dos Santos AC: Cisplatin-induced nephrotoxicity and targets of nephroprotection: An update. Arch Toxicol 86: 1233-1250, 2012.

6. Sánchez-González PD, López-Hernández FJ, López-Novoa JM and Morales AI: An integrative view of the pathophysiological events leading to cisplatin nephrotoxicity. Crit Rev Toxicol 41: 803-821, 2011.

7. Karasawa T and Steyger PS: An integrated view of cisplatin-induced nephrotoxicity and ototoxicity. Toxicol Lett 237: 219-227, 2015.

8. Pabla N and Dong Z: Cisplatin nephrotoxicity: Mechanisms and renoprotective strategies. Kidney Int 73: 994-1007, 2008.

9. Ashkenazi A and Dixit VM: Death receptors: Signaling and modulation. Science 281: 1305-1308, 1998.

10. Zou P, Song J, Jiang B, Pei F, Chen B, Yang X, Liu G and Hu Z: Epigallocatechin-3-gallate protects against cisplatin nephrotoxicity by inhibiting the apoptosis in mouse. Int $\mathrm{J}$ Clin Exp Pathol 7: 4607-4616, 2014.

11. Linkermann A, Himmerkus N, Rölver L, Keyser KA, Steen P Bräsen JH, Bleich M, Kunzendorf U and Krautwald S: Renal tubular Fas ligand mediates fratricide in cisplatin-induced acute kidney failure. Kidney Int 79: 169-178, 2011.

12. Tsuruya K, Ninomiya T, Tokumoto M, Hirakawa M, Masutani K, Taniguchi M, Fukuda K, Kanai H, Kishihara K, Hirakata H and Iida M: Direct involvement of the receptor-mediated apoptotic pathways in cisplatin-induced renal tubular cell death. Kidney Int 63: 72-82, 2003.

13. Tsuruya K, Tokumoto M, Ninomiya T, Hirakawa M, Masutani K, Taniguchi M, Fukuda K, Kanai H, Hirakata $\mathrm{H}$ and Iida M: Antioxidant ameliorates cisplatin-induced renal tubular cell death through inhibition of death receptor-mediated pathways. Am J Physiol Renal Physiol 285: F208-F218, 2003.

14. Scaffidi C, Fulda S, Srinivasan A, Friesen C, Li F, Tomaselli KJ, Debatin KM, Krammer PH and Peter ME: Two CD95 (APO-1/ Fas) signaling pathways. EMBO J 17: 1675-1687, 1998.

15. Wang $X$ and Parrish AR: Loss of $\alpha(E)$-catenin promotes Fas mediated apoptosis in tubular epithelial cells. Apoptosis 20: 921-929, 2015.

16. Luo X, Budihardjo I, Zou H, Slaughter C and Wang X: Bid, a $\mathrm{Bcl} 2$ interacting protein, mediates cytochrome $\mathrm{c}$ release from mitochondria in response to activation of cell surface death receptors. Cell 94: 481-490, 1998.

17. Nogae S, Miyazaki M, Kobayashi N, Saito T, Abe K, Saito H, Nakane PK, Nakanishi Y and Koji T: Induction of apoptosis in ischemia-reperfusion model of mouse kidney: Possible involvement of Fas. J Am Soc Nephrol 9: 620-631, 1998.
18. Schelling JR, Nkemere N, Kopp JB and Cleveland RP: Fas-dependent fratricidal apoptosis is a mechanism of tubular epithelial cell deletion in chronic renal failure. Lab Invest 78: 813-824, 1998.

19. Malhotra A, Bath S and Elbarbry F: An organ system approach to explore the antioxidative, anti-inflammatory and cytoprotective actions of resveratrol. Oxid Med Cell Longev 2015: 803971, 2015.

20. Kitada M and Koya D: Renal protective effects of resveratrol. Oxid Med Cell Longev 2013: 568093, 2013.

21. Catalgol B, Batirel S, Taga Y and Ozer NK: Resveratrol: French paradox revisited. Front Pharmacol 3: 141, 2012.

22. Kanamori H, Takemura G, Goto K, Tsujimoto A, Ogino A, Takeyama T, Kawaguchi T, Watanabe T, Morishita K, Kawasaki M, et al: Resveratrol reverses remodeling in hearts with large, old myocardial infarctions through enhanced autophagy-activating AMP kinase pathway. Am J Pathol 182: 701-713, 2013.

23. Turner RS, Thomas RG, Craft S, van Dyck CH, Mintzer J, Reynolds BA, Brewer JB, Rissman RA, Raman R and Aisen PS; Alzheimer's Disease Cooperative Study: A randomized, double-blind, placebo-controlled trial of resveratrol for Alzheimer disease. Neurology 85: 1383-1391, 2015.

24. Kim DH, Jung YJ, Lee JE, Lee AS, Kang KP, Lee S, Park SK, Han MK, Lee SY, Ramkumar KM, et al: SIRT1 activation by resveratrol ameliorates cisplatin-induced renal injury through deacetylation of p53. Am J Physiol Renal Physiol 301: F427-F435, 2011.

25. Oi N, Jeong CH, Nadas J, Cho YY, Pugliese A, Bode AM and Dong Z: Resveratrol, a red wine polyphenol, suppresses pancreatic cancer by inhibiting leukotriene $\mathrm{A}_{4}$ hydrolase. Cancer Res 70: 9755-9764, 2010.

26. Crandall JP, Oram V, Trandafirescu G, Reid M, Kishore P, Hawkins M, Cohen HW and Barzilai N: Pilot study of resveratrol in older adults with impaired glucose tolerance. J Gerontol A Biol Sci Med Sci 67: 1307-1312, 2012.

27. Palsamy P and Subramanian S: Resveratrol protects diabetic kidney by attenuating hyperglycemia-mediated oxidative stress and renal inflammatory cytokines via Nrf2-Keap1 signaling. Biochim Biophys Acta 1812: 719-731, 2011.

28. Do Amaral CL, Francescato HD, Coimbra TM, Costa RS, Darin JD, Antunes LM and Bianchi Mde L: Resveratrol attenuates cisplatin-induced nephrotoxicity in rats. Arch Toxicol 82: 363-370, 2008.

29. Holthoff JH, Wang Z, Seely KA, Gokden N and Mayeux PR: Resveratrol improves renal microcirculation, protects the tubular epithelium, and prolongs survival in a mouse model of sepsis-induced acute kidney injury. Kidney Int 81: 370-378, 2012.

30. Liu FC, Tsai HI and Yu HP: Organ-Protective effects of red wine extract, resveratrol, in oxidative stress-mediated reperfusion injury. Oxid Med Cell Longev 2015: 568634, 2015.

31. Zhang B, Ramesh G, Norbury CC and Reeves WB: Cisplatin-induced nephrotoxicity is mediated by tumor necrosis factor-alpha produced by renal parenchymal cells. Kidney Int 72 : 37-44, 2007.

32. Li H, Zhu H, Xu CJ and Yuan J: Cleavage of BID by caspase 8 mediates the mitochondrial damage in the Fas pathway of apoptosis. Cell 94: 491-501, 1998.

33. Budihardjo I, Oliver H, Lutter M, Luo X and Wang X: Biochemical pathways of caspase activation during apoptosis. Annu Rev Cell Dev Biol 15: 269-290, 1999.

34. Schug ZT, Gonzalvez F, Houtkooper RH, Vaz FM and Gottlieb E: $\mathrm{BID}$ is cleaved by caspase- 8 within a native complex on the mitochondrial membrane. Cell Death Differ 18: 538-548, 2011.

35. Ott M, Norberg E, Zhivotovsky B and Orrenius S: Mitochondrial targeting of tBid/Bax: A role for the TOM complex? Cell Death Differ 16: 1075-1082, 2009.

36. Korsmeyer SJ, Wei MC, Saito M, Weiler S, Oh KJ and Schlesinger PH: Pro-apoptotic cascade activates BID, which oligomerizes BAK or BAX into pores that result in the release of cytochrome c. Cell Death Differ 7: 1166-1173, 2000. 